\title{
Symptoms of Anxiety and Depression Are Correlates of Angina Pectoris by Recent History and an Ischemia-Positive Treadmill Test in Patients with Documented Coronary Artery Disease in the Pimi Study
}

\author{
Mark W. Ketterer, ${ }^{1}$ Nadine S. Bekkouche, ${ }^{2}$ A. David Goldberg, ${ }^{3}$ Robert P. McMahon, ${ }^{4}$ \\ and David S. Krantz ${ }^{2}$ \\ ${ }^{1}$ Consultation/Liaison Psychiatry, Henry Ford Hospital and Wayne State University, Detroit, MI 48202, USA \\ ${ }^{2}$ Department of Medical \& Clinical Psychology, Uniformed Services University of the Health Sciences, Bethesda, MD 20814, USA \\ ${ }^{3}$ Department of Cardiology, University of Michigan, Ann Arbor, MI 48109, USA \\ ${ }^{4}$ Department of Psychiatry, University of Maryland, Catonsville, MD 21228, USA
}

Correspondence should be addressed to Mark W. Ketterer, mketter1@hfhs.org

Received 24 July 2011; Revised 18 September 2011; Accepted 26 September 2011

Academic Editor: Richard C. Veith

Copyright ( $) 2011$ Mark W. Ketterer et al. This is an open access article distributed under the Creative Commons Attribution License, which permits unrestricted use, distribution, and reproduction in any medium, provided the original work is properly cited.

\begin{abstract}
Objective. We tested the association of specific psychological characteristics in patients having stable coronary disease with the reporting of anginal symptoms during daily activities, and positive exercise testing. Methods. One hundred and ninety-six patients with documented CAD enrolled in the Psychophysiological Investigations of Myocardial Ischemia (PIMI) Study completed an anginal history questionnaire and a battery of psychometric tests. They also underwent standardized exercise treadmill tests. Results. Patients with a recent history of angina were more likely to be female, and had higher Beck Depression $(P=.002)$, State Anxiety $(P=.001)$, Trait Anxiety $(P=.03)$, Harm Avoidance $(P=.04)$ and Muscle Tension $(P=.004)$ scores than patients who had no recent history of angina. Along with several treadmill variables indicating more severe disease state and reduced exercise tolerance, patients who developed angina on a positive treadmill test also displayed higher scores on the Beck Depression Inventory $(P=.003)$ and State Anxiety $(P=.004)$ scales. Conclusions. Several psychological characteristics, and most notably anxiety and depression, are strong correlates of recent angina and angina in the presence of ischemia provoked by treadmill testing.
\end{abstract}

\section{Introduction}

Since the term "angina pectoris" was first coined and recognized as a prodrome to sudden cardiac death $[1,2]$, the causes of chest pain/discomfort have been a topic of investigation and intervention. It is now known that although myocardial ischemia sometimes causes anginal symptoms, the majority of ischemic episodes are silent. Conversely, most episodes of angina-like chest pain in patients known to have coronary artery disease are unaccompanied by measurable ischemia [3]. The factors that determine whether or not angina occurs during ischemia are controversial. Recent studies have identified a variety of physiological correlates, including the type of triggering activity [3], the severity of ischemia or ventricular dysfunction [4], adenosine release [5], endogenous opiate response [6-8], and pain threshold [9].

A variety of psychological characteristics have also been found to be associated with reporting, or denying, anginal symptoms [10-24], or with clinical manifestations of disturbed ischemic pain perception/coping such as treatment seeking delay during acute myocardial infarction [25], silent myocardial infarction $[26,27]$, or unheralded sudden cardiac death [28]. It is difficult to compare these studies because they are not entirely consistent with respect to the psychological variables that were included, or the methods 
and measures used. A principal aim of the Psychophysiological Investigations of Myocardial Ischemia (PIMI) study was to concurrently assess, in a large and well-defined sample of patients with known coronary disease, many of the psychological factors that may be associated with the reporting/denying of anginal symptoms during daily life or on exercise provocation.

On the basis of the earlier studies, we predicted that patients with coronary artery disease who report angina during daily activities, and on a positive exercise treadmill test (ETT) would also report more physical symptoms, and have higher average levels of psychological characteristics or symptoms of such states as anxiety and depression, than patients who develop silent ischemic on the same ETT.

\section{Methods}

Details on the overall protocol of the PIMI Study have been published elsewhere [29-34]. The present study utilizes clinical/demographic data gathered during the recruitment phase of PIMI, results of the baseline qualifying treadmill exam (which was required to be positive for ischemia to achieve eligibility), and psychometric data gathered at a later visit.

2.1. Study Patients. A total of 196 patients (26 females) with stable coronary artery disease (confirmed by positive catheterization and/or past myocardial infarction) who developed myocardial ischemia on a standardized, symptomlimited exercise treadmill test were enrolled in the PIMI Study. All patients were withdrawn from cardiovascular and psychoactive medications three days prior to the qualifying treadmill exam.

2.2. Medical Assessments. Medical history data were obtained from a standardized medical history form. Presence of angina within the last three months was determined using the Rose Questionnaire [35], which was collected as a subscale of the Anginal Syndrome Questionnaire [36]. A screening examination to exclude patients with cognitive impairment or peripheral neuropathy was used for entry to the study.

2.3. Exercise Testing. All patients underwent a standardized, symptom-limited exercise treadmill test using the Asymptomatic Cardiac Ischemia Pilot (ACIP) study ETT protocol [37]. The electrocardiographic criteria for myocardial ischemia were met if either: (a) J point depression $\geq 0.1 \mathrm{~mm}$, and horizontal or $>1 \mathrm{~mm} /$ second downsloping ST segment, and ST-80 depression $\geq 0.15 \mathrm{~mm}$, or (b) ST-80 depression $\geq 0.15 \mathrm{~mm}$ and $\mathrm{T}$ segment sloping upward $>1 \mathrm{~mm} /$ second were present during the treadmill test.

2.4. Psychometric Battery. Subjects were also scheduled to return for psychological testing within three months after completing the ETT. The psychometric battery consisted of seven questionnaires administered by study personnel; the Beck Depression Inventory (BDI)_a 21-item measure of the severity of depressive symptoms [38]; the StateTrait Anxiety Inventory (STAI) - a measure of acute and chronic anxiety [39]; a modified version of the Autonomic Perception Questionnaire (MAPQ) which included subscales on Autonomic Perception and Muscle Tension [40]; the Toronto Alexithymia Scale (TAS) - a 26-item measure of awareness of the external environment to the exclusion of self-awareness [41]; the 24-item Anger Expression Scale (AX) which includes subscales for Anger Out (AXOUT) to assess overtly expressed anger, Anger In (AXIN) to assess unexpressed anger, and Anger Control (AXCON) to assess efforts to suppress the overt expression of anger [42]; the Cook-Medley Hostility Scale (CMS) - a 50-item measure of hostile attitudes [43]; the Tridimensional Personality Questionnaire (TPQ) which includes the 35-item Harm Avoidance Scale (HA) to assess the tendency to avoid stimuli perceived to be potentially harmful or painful, the 40 -item Novelty Seeking Scale (NA) to assess the tendency to respond strongly to novel stimuli and to cues for relief from aversive stimulation, the 24-item Reward Dependence Scale (RD) to assess the tendency to respond strongly to social approval and other sources of positive reinforcement [44].

2.5. Statistical Analysis. The normal scores rank test was used instead of the two-sample $t$-test to compare continuously distributed variables in order to protect against the effects of outliers and nonnormal distributions $[45,46]$. Pearson's chisquare test was used to test for association between discrete variables. In order to conduct a Stepwise Cox Regression of angina on the treadmill test using risk factors known to covary with angina (Sex and History of Hypertension-[22]) as well as two indices of disease severity (Time to Initial ST Segment Depression and Rate-Pressure Product at Initial ST segment depression) and State Anxiety and Depression, patients without angina were scored as having Angina onset at one minute past the maximum number of minutes any patient stayed on the test. A Stepwise Logistic Regression using the same control variables was used to conduct the same analysis for angina by history.

\section{Results}

3.1. History of Angina. Seventy patients had a history of angina by Rose criteria during the three months prior to entering the study, while 126 had no such history. Table 1 displays comparisons for the demographic and medical characteristics between patients who reported a history of anginal symptoms during the three months prior to the study, and those who were asymptomatic during this period. Patients with a recent history of angina were more likely to be female but were otherwise not different on clinical/demographic variables. The patients with recent angina did, however, tend to score higher on the Beck Depression Inventory, the State Anxiety Scale, the Trait Anxiety Scale, and the Harm Avoidance and Muscle Tension subscales. See Table 1. In the Stepwise Logistic Regression (chi-square $=15.1$, $\mathrm{df}=2, P=$ $0.001)$, only time to ST segment depression $(B=-.107)$ and 
TABle 1: Demographic, medical, and psychological characteristics of patients with versus without a recent (past three months) history of anginal symptoms.

\begin{tabular}{|c|c|c|c|}
\hline \multirow{3}{*}{ Variable } & \multicolumn{2}{|c|}{ Recent anginal symptoms } & \multirow{3}{*}{$P$} \\
\hline & $\begin{array}{l}\text { Present } \\
(N=70)\end{array}$ & $\begin{array}{c}\text { Absent } \\
(N=126)\end{array}$ & \\
\hline & & $\%$ & \\
\hline Male & $80 \%$ & $90.5 \%$ & .04 \\
\hline Caucasian & $82.9 \%$ & $89.7 \%$ & .17 \\
\hline History of Diabetes & $15.7 \%$ & $14.3 \%$ & .79 \\
\hline $\begin{array}{l}\text { History of Myocardial } \\
\text { Infarction }\end{array}$ & $41.4 \%$ & $42.9 \%$ & .85 \\
\hline History of Hypertension & $54.3 \%$ & $43.7 \%$ & .15 \\
\hline History of PTCA & $35.7 \%$ & $34.9 \%$ & .91 \\
\hline Variable & $\mathrm{M}(\mathrm{SD})$ & $\mathrm{M}(\mathrm{SD})$ & $P$ \\
\hline Age & $61.4(8.7)$ & $63.1(8.2)$ & .17 \\
\hline $\begin{array}{l}\text { Beck Depression } \\
\text { Inventory }\end{array}$ & $7.3(5.7)$ & $5.2(5.7)$ & .002 \\
\hline State Anxiety & $34.5(9.8)$ & $29.8(9.5)$ & .001 \\
\hline Trait Anxiety & $34.3(8.7)$ & $31.6(8.7)$ & .03 \\
\hline Novelty Seeking & $16.5(6.2)$ & $16.5(5.6)$ & .97 \\
\hline Harm Avoidance: & $13.0(6.2)$ & $11.1(7.3)$ & .04 \\
\hline Reward Dependence: & $15.8(4.4)$ & $15.8(3.7)$ & .98 \\
\hline $\begin{array}{l}\text { Cook-Medley Hostility } \\
\text { Scale }\end{array}$ & $19.9(8.0)$ & $20.1(8.7)$ & .97 \\
\hline Anger Out & $13.9(3.4)$ & $13.8(3.8)$ & .69 \\
\hline Anger In & $15.0(3.3)$ & $15.4(4.1)$ & .69 \\
\hline Anger Control & $25.5(5.3)$ & $26.0(4.8)$ & .71 \\
\hline Anger Expression & $19.5(9.4)$ & $19.3(9.4)$ & .93 \\
\hline Autonomic Perception & $71.9(32.3)$ & $64.2(31.7)$ & .09 \\
\hline Muscle Tension & $32.1(17.3)$ & $25.2(18.4)$ & .004 \\
\hline $\begin{array}{l}\text { Toronto Alexithymia } \\
\text { Scale }\end{array}$ & $64.7(12.0)$ & $63.2(12.4)$ & .47 \\
\hline
\end{tabular}

State Anxiety $(B=.055)$ survived as unique predictors of angina by history.

3.2. Exercise Treadmill Test. Ninety patients developed angina during the qualifying treadmill test, while $106 \mathrm{did}$ not. As shown in Table 2, there were no differences on clinical/demographic characteristics between patients experiencing angina on the ischemia-positive ETT and those who did not. However, patients with angina had evidence of more severe disease (shorter Time to $1 \mathrm{~mm}$ ST Depression and lower Rate-Pressure Product at ST Onset) and diminished exercise tolerance (less Total ETT Duration, Time to Ends of Test from ST Onset, and lower Rate-Pressure Product at Peak Exercise). Patients who reported angina on the positive ETT displayed higher average scores on the Beck Depression Inventory and the State Anxiety Scale. In the Stepwise Cox Regression (chi-square $=17.2, \mathrm{df}=2, P<0.001$ ), only time to ST segment depression $(B=-.165)$ and Depression $(B=0.051)$ retained unique variance.

\section{Discussion}

Our results have demonstrated that patients with angina have a higher frequency of depressive/anxious symptoms. The relationship between angina pectoris in daily life and psychological characteristics has become a topic of some interest in recent years. There have also been several studies examining psychological characteristics in coronary patients with, and without, angina in response to treadmill exercise testing. These studies, while only correlational in nature, are important for several reasons. First they may provide some insight into clinical situations in which angina as a warning sign seems to fail, such as excessive treatment seeking delay during acute myocardial infarction [25], diagnosis of acute myocardial infarction [47], silent myocardial infarction [26, 27], and unheralded sudden cardiac death [28]. Second, they may also carry some implications for controlling angina if the psychological correlates are found to be the cause, rather than the result, of the symptoms. Indeed, several studies already indicate that treatment of emotional distress with cognitive-behavioral therapy reduces angina symptoms [48-57]. Efficacy of psychopharmacological interventions for anxiety/depression in reducing angina in this population is not yet demonstrated, but such studies are urgently needed.

The present study is unique in that it permits comparisons, within a single sample, of patients both with and without a recent history of angina, and those who are asymptomatic or not on an ischemia-positive treadmill test. The size of the present sample is large in comparison to other studies, the psychometric assessment is broad and comprehensive, and the well-defined baseline characteristics of the sample are also unique. The study may be limited, however, by the fact that the psychometric tests were not administered on the same day as the treadmill exam. This may have weakened the correlations between psychometric tests and angina, since the patient's mood state may have changed in the intervening interval.

The State Anxiety Scale and Beck Depression Inventory were strongly associated with Angina both by recent history, and on a positive treadmill test. Trait Anxiety, Harm Avoidance, and Muscle Tension were also found to be associated with angina by recent history. Alexithymia was unrelated to the presence or absence of angina. The adverse impact of alexithymia on treatment-seeking behavior [25] may not be directly mediated by symptom burden. Anger was also unrelated to the presence or absence of angina.

This may appear paradoxical to those who assume that ischemia is the principle or only cause of angina in the CAD patient, since even artificial, contrived, and brief angerevoking situations are known to cause ischemia in about half of patients with documented CAD [58]. We interpret this as indicating that the psychological causes of ischemia and angina may be distinct.

Several studies have now reported that patients who experience angina are more aware of physical symptoms in general than are those with silent ischemia [59]. Our results raise the question of whether somatic hypersensitivity, a well-known and common manifestation of depression and anxiety [60], might not determine whether patients 
TABle 2: Demographic and medical characteristics, ETT parameters and psychological test scores by presence or absence of anginal symptoms during the positive exercise treadmill test.

\begin{tabular}{|c|c|c|c|}
\hline \multirow{3}{*}{ Variable } & \multicolumn{2}{|c|}{ Type of ischemia during ETT } & \multirow{4}{*}{$P$} \\
\hline & Symptomatic & Silent & \\
\hline & $(N=90)$ & $(N=106)$ & \\
\hline & $\%$ & $\%$ & \\
\hline Male & 87 & 87 & .98 \\
\hline Caucasian & 88 & 87 & .84 \\
\hline History of Diabetes & 13 & 16 & .60 \\
\hline History of Myocardial Infarction & 44 & 41 & .58 \\
\hline History of Hypertension & 53 & 42 & .13 \\
\hline \multirow[t]{3}{*}{ History of PTCA } & 31 & 39 & .27 \\
\hline & $\mathrm{M}$ & $\mathrm{M}$ & \\
\hline & $(\mathrm{SD})$ & $(\mathrm{SD})$ & \\
\hline Age & $62.5(8.7)$ & $62.5(8.2)$ & .91 \\
\hline Total ETT Duration (mins) & $6.9(2.7)$ & $8.4(3.0)$ & $<.001$ \\
\hline Time to $1 \mathrm{~mm}$ ST Depression & $4.8(2.3)$ & $5.9(3.4)$ & .008 \\
\hline Time to End of Test from ST Onset & $2.0(1.5)$ & $2.5(2.6)$ & .03 \\
\hline Maximum St Depression (mm) & $1.9(0.6)$ & $2.1(0.6)$ & .04 \\
\hline Rate-Pressure Product at Onset of ST Depression (/1000) & $21.8(4.7)$ & $23.7(5.6)$ & .02 \\
\hline Rate-Pressure Product at Peak Exercise (/1000) & $23.6(4.5)$ & $26.4(5.7)$ & $<.001$ \\
\hline Peak Borg Exertion Score & 16.1 & 16.2 & .72 \\
\hline Peak SBP & $177.8(21.3)$ & $184.6(27.7)$ & .07 \\
\hline Peak-Resting SBP & $36.6(19.0)$ & $42.2(27.2)$ & .07 \\
\hline Beck Depression Inventory & $7.3(6.9)$ & $4.7(4.4)$ & .003 \\
\hline State Anxiety & $33.9(10.2)$ & $29.5(9.0)$ & .004 \\
\hline Trait Anxiety & $34.0(9.4)$ & $31.3(8.1)$ & .07 \\
\hline Modified Autonomic Perception & $68.6(32.6)$ & $65.5(31.8)$ & .49 \\
\hline Muscle Tension Perception & $30.0(18.9)$ & $25.8(17.6)$ & .09 \\
\hline Toronto Alexithymia Scale & $64.7(13.3)$ & $63.0(11.3)$ & .27 \\
\hline Anger Out & $13.9(3.4)$ & $13.8(3.7)$ & .79 \\
\hline Anger In & $15.4(3.8)$ & $15.1(3.9)$ & .56 \\
\hline Anger Control & $25.5(5.3)$ & $26.1(4.7)$ & .64 \\
\hline Anger Expression & $19.9(9.4)$ & $18.9(9.4)$ & .53 \\
\hline Cook-Medley Hostility Scale & $21.0(8.2)$ & $19.2(8.5)$ & .09 \\
\hline Harm Avoidance & $12.8(7.4)$ & $11.0(6.6)$ & .11 \\
\hline Novelty Seeking & $16.1(5.7)$ & $16.9(5.9)$ & .40 \\
\hline Reward Dependence & $15.4(4.1)$ & $16.1(3.8)$ & .18 \\
\hline
\end{tabular}

with coronary artery disease experience or report angina. A number of chronic pain conditions are known to lower pain thresholds $[61,62]$. This suggests that treatment of anxiety and depression might help to control anginal symptoms (and thus treatment costs) in at least some patients. Healthcare costs are known to be elevated in emotionally distressed CAD patients [63-69]. While these increased costs may be due to more rapid disease progression in some patients [70-73], it also seems likely that greater symptom burden, including other symptoms thought to be caused by ischemic heart disease $[22,74]$ would increase treatment-seeking for patients, and testing/admission rates for physicians.
In several studies, treatment of distress has been found to reduce utilization and costs [21,75-77]. Aggressive, anginatargeted clinical trials that also evaluate impact on utilization, and trials using modern psychopharmacological agents, are needed.

\section{Conclusions}

Patients with known coronary artery disease who report experiencing angina, either by recent history or an ischemiapositive exercise treadmill test, are more likely to report anxious and depressive symptoms. Heightened awareness of 
physical symptoms, and possibly higher levels of anxiety and depression, differentiate between patients who report angina and those who do not. This raises the question of whether treatment of anxiety and depression may help to diminish angina.

\section{Disclosure}

Portions of this study were presented at the annual meeting of the American Psychosomatic Society, Williamsburg, VA, $3 / 10 / 96$.

\section{Acknowledgments}

The Psychophysiological Investigations of Myocardial Ischemia (PIMI) was supported by contracts HV18114, HV18119, HV18120, HV18121, and HV 28127 from the National Heart, Lung and Blood Institute. Support of electrocardiographic data collection was provided in part by Applied Cardiac Systems, Laguna Hills, California; Marquette Electronics, Milwaukee, Wisconsin; Quinton Instruments, Seattle, Washington; and Mortara Instruments, Milwaukee, Wisconsin. Dinamap equipment was provided by Critikon, Inc., a Johnson \& Johnson Company. Michael Eddy, University of Pittsburgh, and Richard Lutz, University of North Carolina, provided Stroop Test software, and Dr. Willkiam Maixner, University of North Carolina, provided software and design of the Marstock Sensort Threshold Test. Dr. Kathleen Light provided role play scenarios for the Speech Test. Some centers had partial support from General Clinical Research Center grants. A list of participating centers and investigators appears in Lumley et al. [26].

\section{References}

[1] W. Heberden, "Some account of a disorder of the breast," Medical Transactions of the College of Physicians, vol. 2, pp. 5967, 1772.

[2] P. Kligfieid and K. Filutowski, “"Dr. Anonymous” unmasked: resolution of an eighteenth century mystery in the history of coronary artery disease," American Journal of Cardiology, vol. 75, no. 16, p. 1166, 1995.

[3] D. S. Krantz, S. M. Hedges, F. H. Gabbay et al., "Triggers of angina and ST-segment depression in ambulatory patients with coronary artery disease: evidence for an upcoupling of angina and ischemia," American Heart Journal, vol. 128, no. 4, pp. 703-712, 1994.

[4] P. Nihoyannopoulos, A. Marsonis, J. Joshi, G. Athanassopoulos, and C. M. Oakley, "Magnitude of myocardial dysfunction is greater in painful than in painless myocardial ischemia: an exercise echocardiographic study," Journal of the American College of Cardiology, vol. 25, no. 7, pp. 1507-1512, 1995.

[5] F. Crea, G. Pupita, A. R. Galassi et al., "Role of adenosine in pathogenesis of anginal pain," Circulation, vol. 81, no. 1, pp. 164-172, 1990.

[6] P. F. Miller, K. C. Light, E. E. Bragdon et al., "Beta-endorphin response to exercise and mental stress in patients with ischemic heart disease," Journal of Psychosomatic Research, vol. 37, no. 5, pp. 455-465, 1993.
[7] A. C. Sena, W. Maixner, M. N. Ballenger, M. C. Herbst, G. Koch, and D. S. Sheps, "The relationship between plasma $\beta$-endorphin, opioid receptor activity, and silent myocardial ischemia," Clinical Journal of Pain, vol. 8, no. 4, pp. 307-316, 1992.

[8] D. S Sheps, M. N. Ballenger, G. E. de Gent et al., "Psychophysiological responses to a speech stressor: correlation of plasma beta-endorphin levels at rest and after psychological stress with thermally measured pain threshold in patients with coronary artery disease," Journal of the American College of Cardiology, vol. 25, pp. 1499-1503, 1995.

[9] H. Hikita, A. Kurita, B. Takase et al., "Usefulness of plasma beta-endorphin level, pain threshold and autonomic function in assessing silent myocardial ischemia in patients with and without diabetes mellitus," American Journal of Cardiology, vol. 72, no. 2, pp. 140-143, 1993.

[10] R. F. Davies, W. Linden, H. Habibi et al., "Relative importance of psychologic traits and severity of ischemia in causing angina during treadmill exercise," Journal of the American College of Cardiology, vol. 21, no. 2, pp. 331-336, 1993.

[11] K. E. Freedland, R. M. Carney, R. J. Krone et al., "Psychological factors in silent myocardial ischemia," Psychosomatic Medicine, vol. 53, no. 1, pp. 13-24, 1991.

[12] K. E. Freedland, R. M. Carney, R. J. Krone, N. B. Case, and R. B. Case, "Psychological determinants of anginal pain perception during exercise testing of stable patients after recovery from acute myocardial infarction or unstable angina pectoris," American Journal of Cardiology, vol. 77, no. 1, pp. $1-4,1996$.

[13] K. C. Light, M. C. Herbst, E. E. Bragdon et al., "Depression and Type A behavior pattern in patients with coronary artery disease: relationships to painful versus silent myocardial ischemia and $\beta$ - endorphin responses during exercise," Psychosomatic Medicine, vol. 53, no. 6, pp. 669-683, 1991.

[14] C. D. Jenkins, B. A. Stanton, M. D. Klein, J. A. Savageau, and D. E. Harken, "Correlates of angina pectoris among men awaiting coronary by-pass surgery," Psychosomatic Medicine, vol. 45, no. 2, pp. 141-153, 1983.

[15] P. T. Costa, A. B. Zonderman, B. T. Engel, W. F. Baile, D. L. Brimlow, and J. Brinker, "The relation of chest pain symptoms to angiographic findings of coronary artery stenosis and neuroticism," Psychosomatic Medicine, vol. 47, no. 3, pp. 285-293, 1985.

[16] A. Bengtson, J. Herlitz, T. Karlsson, and A. Hjalmarson, "Distress correlates with the degree of chest pain: a description of patients awaiting revascularisation," Heart, vol. 75 , no. 3 , pp. 257-260, 1996.

[17] U. Verthein and T. Köhler, "The correlation between everyday stress and angina pectoris: a longitudinal study," Journal of Psychosomatic Research, vol. 43, no. 3, pp. 241-245, 1997.

[18] K. H. Ladwig, G. Röll, G. Breithardt, and M. Borggrefe, "Extracardiac contributions to chest pain perception in patients 6 months after acute myocardial infarction," American Heart Journal, vol. 137, no. 3, pp. 528-534, 1999.

[19] J. Barry, A. P. Selwyn, E. G. Nabel et al., "Frequency of STsegment depression produced by mental stress in stable angina pectoris from coronary artery disease," American Journal of Cardiology, vol. 61, no. 13, pp. 989-993, 1988.

[20] M. W. Ketterer, J. Huffman, M. A. Lumley et al., "Fiveyear follow-up for adverse outcomes in males with at least minimally positive angiograms: importance of "denial" in 
assessing psychosocial risk factors," Journal of Psychosomatic Research, vol. 44, no. 2, pp. 241-250, 1998.

[21] M. W. Ketterer, F. Fitzgerald, S. Keteyian et al., "Chest Pain and the Treatment of Psychosocial/Emotional Distress in CAD Patients," Journal of Behavioral Medicine, vol. 23, no. 5, pp. 437-450, 2000.

[22] M. W. Ketterer, W. Knysz, S. J. Keteyian et al., "Cardiovascular symptoms in coronary-artery disease patients are strongly correlated with emotional distress," Psychosomatics, vol. 49, no. 3, pp. 230-234, 2008.

[23] T. W. Smith, M. J. Follick, and K. S. Korr, "Anger, neuroticism, type A behaviour and the experience of angina," British Journal of Medical Psychology, vol. 57, no. 3, pp. 249-252, 1984.

[24] T. Torosian, M. A. Lumley, S. D. Pickard, and M. W. Ketterer, "Silent versus symptomatic myocardial ischemia: the role of psychological and medical factors," Health Psychology, vol. 16, no. 2, pp. 123-130, 1997.

[25] L. W. Kenyon, M. W. Ketterer, M. Gheorghiade, and S. Goldstein, "Psychological factors related to prehospital delay during acute myocardial infarction," Circulation, vol. 84, no. 5, pp. 1969-1976, 1991.

[26] M. A. Lumley, T. Torosian, L. L. Rowland, M. W. Ketterer, and S. D. Pickard, "Correlates of unrecognized acute myocardial infarction detected via perfusion imaging," American Journal of Cardiology, vol. 79, no. 9, pp. 1170-1173, 1997.

[27] M. E. Theisen, S. E. MacNeill, M. A. Lumley, M. W. Ketterer, A. D. Goldberg, and S. Borzak, "Psychosocial factors related to unrecognized acute myocardial infarction," American Journal of Cardiology, vol. 75, no. 17, pp. 1211-1213, 1995.

[28] S. Goldstein, "Toward a new understanding of the mechanism and prevention of sudden death in coronary heart disease," Circulation, vol. 82, no. 1, pp. 284-288, 1990.

[29] L. C. Becker, C. J. Pepine, R. Bonsall et al., "Left ventricular, peripheral vascular, and neurohumoral responses to mental stress in normal middle-aged men and women: reference group for the Psychophysiological Investigations of Myocardial Ischemia (PIMI) study," Circulation, vol. 94, no. 11, pp. 2768-2777, 1996.

[30] R. M. Carney, R. P. McMahon, K. E. Freedland et al., "Reproducibility of mental stress-induced myocardial ischemia in the psychophysiological investigations of myocardial ischemia (PIMI)," Psychosomatic Medicine, vol. 60, no. 1, pp. 64-70, 1998.

[31] A. D. Goldberg, L. C. Becker, R. Bonsall et al., "Ischemic, hemodynamic, and neurohormonal responses to mental and exercise stress: experience from the Psychophysiological Investigations of Myocardial Ischemia study (PIMI)," Circulation, vol. 94, no. 10, pp. 2402-2409, 1996.

[32] P. G. Kaufmann, R. P. McMahon, L. C. Becker et al., "The psychophysiological investigations of myocardial ischemia (PIMI) study: objective, methods, and variability of measures," Psychosomatic Medicine, vol. 60, no. 1, pp. 56-63, 1998.

[33] D. S. Sheps, R. P. McMahon, K. C. Light et al., "Low hot pain threshold predicts shorter time to exercise-induced angina: results from the psychophysiological investigations of myocardial ischemia (PIMI) study," Journal of the American College of Cardiology, vol. 33, no. 7, pp. 1855-1862, 1999.

[34] P. H. Stone, D. S. Krantz, R. P. McMahon et al., " Relationship among mental stress induced ischemia and ischemia during daily life and during exercise: the psychophysiological investigations of myocardal ischemia (pimi) study," Journal of the
American College of Cardiology, vol. 33, no. 6, pp. 1484-1484, 1999.

[35] G. A. Rose, "The diagnosis of ischaemic heart pain and intermittent claudication in field surveys," Bulletin of the World Health Organization, vol. 27, pp. 645-658, 1962.

[36] A. F. Greene, D. D. Schocken, and C. D. Spielberger, "Selfreport of chest pain symptoms and coronary artery disease in patients undergoing angiography," Pain, vol. 47, no. 3, pp. 319-324, 1991.

[37] B. Tamesis, A. Stelken, S. Byers et al., "Comparison of the asymptomatic cardiac ischemia pilot and modified asymptomatic cardiac ischemia pilot versus Bruce and Cornell exercise protocols," American Journal of Cardiology, vol. 72, no. 9, pp. 715-720, 1993.

[38] A. T. Beck, C. H. Ward, M. Mendelsohn, J. Mock, and J. Erbaugh, "An inventory for measuring depression," Archives of general psychiatry, vol. 4, pp. 561-571, 1961.

[39] C. D. Spielberger, R. L. Gorsuch, and R. D. Lushene, Manual for the State-Trait Anxiety Inventory, Consulting Psychologists Press, Palo Alto, Calif, USA, 1970.

[40] G. Mandler, J. M. Mandler, and E. T. Uviller, "Autonomic feedback: The perception of autonomic activity," Journal of Abnormal and Social Psychology, vol. 56, no. 3, pp. 367-373, 1958.

[41] G. J. Taylor, R. M. Bagby, D. P. Ryan, J. D.A. Parker, K. F. Doody, and P. Keefe, "Criterion validity of the Toronto Alexithymia Scale," Psychosomatic Medicine, vol. 50, no. 5, pp. 500-509, 1988.

[42] C. D. Spielberger, E. H. Johnson, S. F. Russell, R. J. Crane, G. A. Jacobs, and T. J. Worden, "The experience and expression of anger: construction and validation of an anger expression scale," in Anger and Hostility in Cardiovascular and Behavioral Disorders, M. A. Chesney and R. H. Rosenman, Eds., McGrawHill, New York, NY, USA, 1985.

[43] W. W. Cook and D. M. Medley, "Proposed hostility and Pharisaic-virtue scales for the MMPI," Journal of Applied Psychology, vol. 38, no. 6, pp. 414-418, 1954.

[44] C. R. Cloninger, "A systematic method for clinical description and classification of personality variants," Archives of General Psychiatry, vol. 44, no. 6, pp. 573-588, 1987.

[45] W. J. Conover, Practical Nonparametric Statistics, John Wiley \& Sons, New York, NY, USA, 2nd edition, 1980.

[46] D. G. Kleinbaum, L. L. Kupper, and H. Morganstern, Epidemiological Research: Principles and Quantitative Methods, Van Nostrand Reinhold, New York, NY, USA, 1982.

[47] M. Edwards, A. M. Chang, A. C. Matsuura, M. Green, J. M. Robey, and J. E. Hollander, "Relationship between painseverity and outcomes in patients presenting with potential acute coronary syndromes," Annals of Emergency Medicine. In press.

[48] J. A. Blumenthal, W. Jiang, M. A. Babyak et al., "Stress management and exercise training in cardiac patients with myocardial ischemia: effects on prognosis and evaluation of mechanisms," Archives of Internal Medicine, vol. 157, no. 19, pp. 2213-2223, 1997.

[49] B. Lewin, "The psychological and behavioral management of angina," Journal of Psychosomatic Research, vol. 43, no. 5, pp. 453-462, 1997.

[50] R. Collins, D. Ornish, L. Scherwitz et al., "Are comprehensive lifestyle changes feasible and clinically beneficial?" Psychosomatic Medicine, vol. 58, p. 85, 1996. 
[51] T. J. Payne, C. A. Johnson, D. B. Penzien et al., "Chest pain self-management training for patients with coronary artery disease," Journal of Psychosomatic Research, vol. 38, no. 5, pp. 409-418, 1994.

[52] M. S. Rapp and M. R. Thomas, "Alleviation of angina pectoris following systematic desensitization," Canadian Psychiatric Association Journal, vol. 20, no. 1, p. 96, 1975.

[53] C. H. Hartman, "Response of anginal pain to handwarming," Biofeedback \& Self-Reg, vol. 4, pp. 355-357, 1979.

[54] D. W. Johnston and C. R. Lo, "The effects of cardiovascular feedback and relaxation on angina pectoris," Behavioural Psychotherapy, vol. 11, pp. 257-264, 1983.

[55] B. Amarosa-Tupler, J. T. Tapp, and R. V. Carida, "Stress management through relaxation and imagery in the treatment of angina pectoris," Journal of Cardiopulmonary Rehabilitation, vol. 9, no. 9, pp. 348-355, 1989.

[56] C. Bundy, D. Carroll, L. Wallace, and R. Nagle, "Psychological treatment of chronic stable angina pectoris," Psychology \& Health, vol. 10, pp. 69-77, 1994.

[57] E. Jonsbu, T. Dammen, G. Morken, T. Moum, and E. W. Martinsen, "Short-term cognitive behavioral therapy for noncardiac chest pain and benign palpitations: a randomized controlled trial," Journal of Psychosomatic Research, vol. 70, pp. 117-123, 2011.

[58] M. W. Ketterer, K. E. Freedland, D. S. Krantz et al., "Psychological correlates of mental stress-induced ischemia in the laboratory: the psychophysiological investigation of myocardial ischemia (PIMI) study," Journal of Health Psychology, vol. 5, no. 1, pp. 75-85, 2000.

[59] A. J. Barsky, B. Hochstrasser, N. A. Coles, J. Zisfein, C. O'Donnell, and K. A. Eagle, "Silent myocardial ischemia. Is the person or the event silent?" Journal of the American Medical Association, vol. 264, no. 9, pp. 1132-1135, 1988.

[60] M. Swartz, R. Landerman, L. K. George, D. G. Blazer, and J. Escobar, "Somatization disorder," in Psychiatric Disorders in America: The Epidemiological Catchment Area Study, L. N. Robins and D. A. Regier, Eds., pp. 220-257, The Free Press, New York, NY, USA, 1991.

[61] A. J. Bouckoms and T. P. Hackett, "Pain patients," in Massachusetts General Hospital Handbook of General Hospital Psychiatry, N. H. Cassem, T. A. Stern, J. F. Rosenbaum, and M. S. Jellinek, Eds., pp. 367-414, Mosby, St. Louis, Mo, USA, 1997.

[62] I. Pilowsky, "Affective disorders and pain," in Proceedings of the 5th World Congress on Pain, R. Dubner, G. F. Gebhart, and M. R. Bond, Eds., Elsevier, Amsterdam, The Netherland, 1988.

[63] T. G. Allison, D. E. Williams, T. D. Miller et al., "Medical and economic costs of psychologic distress in patients with coronary artery disease," Mayo Clinic Proceedings, vol. 70, pp. 734-742, 1995.

[64] K. W. Davidson, "Dose-response relations between hostility reductions and cardiac related hospitalizations ," Psychosomatic Medicine, vol. 47, pp. 285-293, 2000.

[65] N. Frasure-Smith and F. Lesperance, "Depression and anxiety increase physician costs during the first post-MI year," Psychosomatic Medicine, vol. 60, p. 99, 1998.

[66] J. C. Huffman, F. A. Smith, M. A. Blais, J. L. Januzzi, and G. L. Fricchione, "Anxiety, independent of depressive symptoms, is associated with in-hospital cardiac complications after acute myocardial infarction," Journal of Psychosomatic Research, vol. 65, no. 6, pp. 557-563, 2008.
[67] M. W. Ketterer, W. Knysz, A. Khandelwal, S. J. Keteyian, A. Farha, and S. Deveshwar, "Healthcare utilization and emotional distress in coronary artery disease patients," Psychosomatics, vol. 51, no. 4, pp. 297-301, 2010.

[68] E. Mumford, H. J. Schlesinger, and G. V. Glass, "The effects of psychological intervention on recovery from surgery and heart attacks: an analysis of the literature," American Journal of Public Health, vol. 72, no. 2, pp. 141-151, 1982.

[69] D. Thompson, T. R. Hylan, W. McMullen et al., "Predictors of a medical-offset effect among patients receiving antidepressant therapy," American Journal of Psychiatry, vol. 155, no. 6, pp. 824-827, 1998.

[70] G. Burell, "Group psychotherapy in project new life: treatment of coronary-prone behaviors for patients who have had coronary artery bypass graft surgery," in Heart \& Mind: The Practice of Cardiasc Psychology, R. Allan and S. Scheidt, Eds., pp. 291-312, American Psychological Association, Wash, USA, 1996.

[71] M. Friedman, C. E. Thoresen, and J. J. Gill, "Alteration of type A behavior and its effect on cardiac recurrences in post myocardial infarction patients: summary results of the recurrent coronary prevention project," American Heart Journal, vol. 112, no. 4, pp. 653-665, 1986.

[72] M. Friedman, L. H. Powell, C. E. Thoresen et al., "Effect of discontinuance of type A behavioral counseling on type A behavior and cardiac recurrence rate of post myocardial infarction patients," American Heart Journal, vol. 114, no. 3, pp. 483-490, 1987.

[73] M. Gulliksson, G. Burell, B. Vessby, L. Lundin, H. Toss, and K. Svärdsudd, "Randomized controlled trial of cognitive behavioral therapy vs standard treatment to prevent recurrent cardiovascular events in patients with coronary heart disease," Archives of Internal Medicine, vol. 171, no. 2, pp. 134-140, 2011.

[74] A. Bunevicius, A. Stankus, J. Brozaitiene, S. S. Girdler, and R. Bunevicius, "Relationship of fatigue and exercise capacity with emotional and physical state in patients with coronary artery disease admitted for rehabilitation program," American Heart Journal, vol. 162, no. 2, pp. 310-316, 2011.

[75] J. L. Black, T. G. Allison, D. E. Williams, T. A. Rummans, and G. T. Gau, "Effect of intervention for psychological distress on rehospitalization rates in cardiac rehabilitation patients," Psychosomatics, vol. 39, no. 2, pp. 134-143, 1998.

[76] J. A. Blumenthal, M. Babyak, J. Wei et al., "Usefulness of psychosocial treatment of mental stress-induced myocardial ischemia in men," American Journal of Cardiology, vol. 89, no. 2, pp. 164-168, 2002.

[77] B. Lewin, I. H. Robertson, E. L. Cay, J. B. Irving, and M. Campbell, "Effects of self-help post-myocardial-infarction rehabilitation on psychological adjustment and use of health services," Lancet, vol. 339, no. 8800, pp. 1036-1040, 1992. 


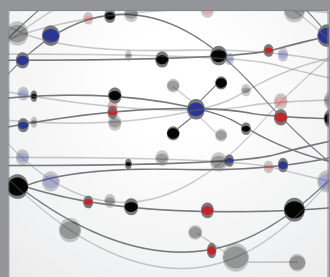

The Scientific World Journal
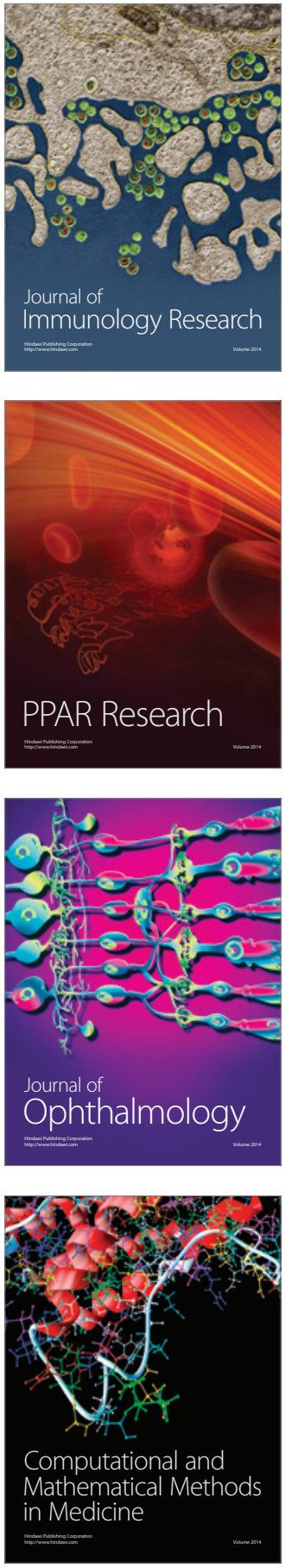

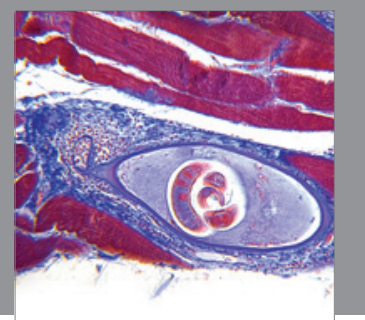

Gastroenterology

Research and Practice
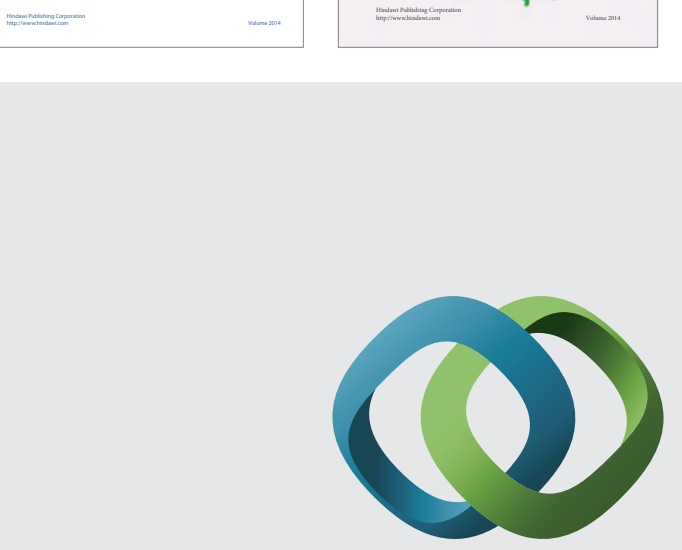

\section{Hindawi}

Submit your manuscripts at

http://www.hindawi.com
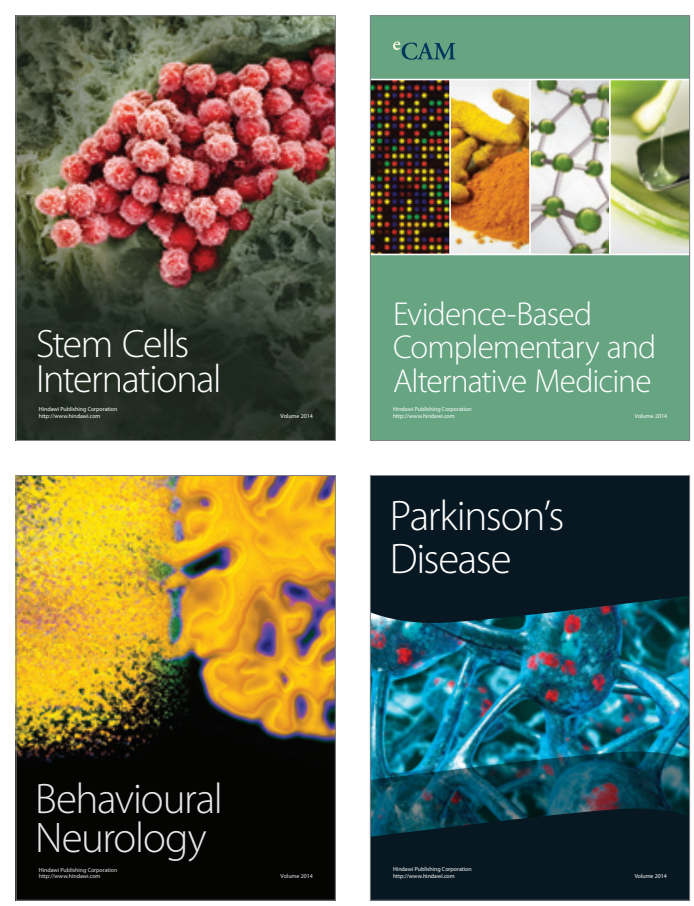

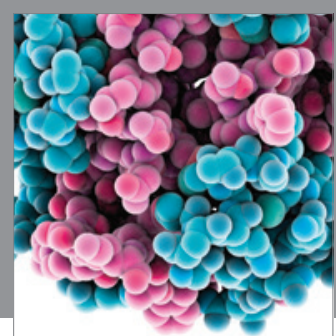

Journal of
Diabetes Research

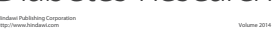

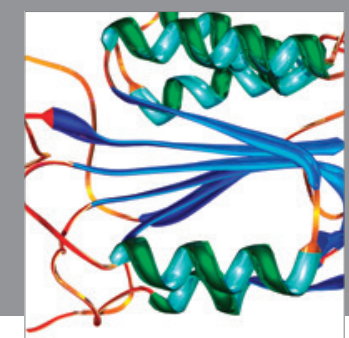

Disease Markers
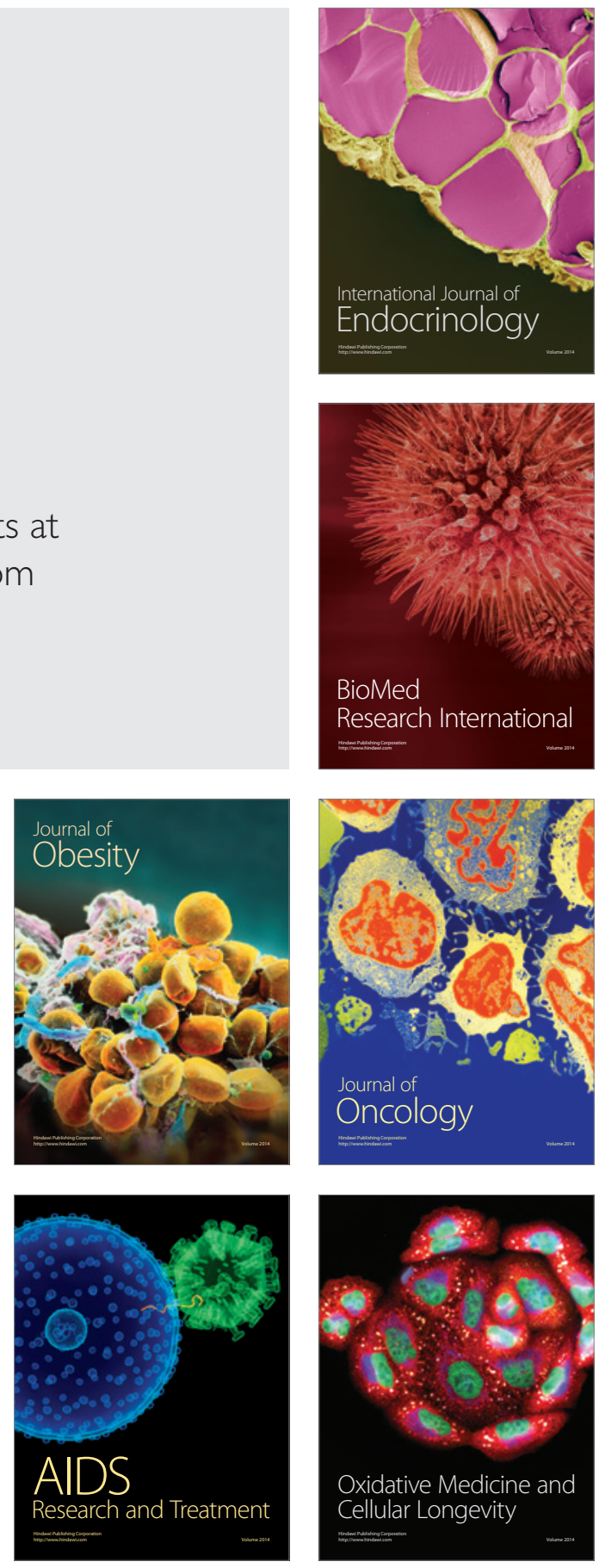